\title{
Rediseño del prototipo de sistema programable para prevenir el robo de bicicletas en Oaxaca
}

\author{
Redesign of programmable system prototype to prevent bicycle theft in Oaxaca \\ MORALES-HERNÁNDEZ, Maricela†*, MARTÍNEZ-HERNÁNDEZ, Gabriela, GUZMÁN-SALAS, \\ León y RAFAEL-PÉREZ, Eva
}

Tecnológico Nacional de México, Campus Oaxaca. Avenida Ing. Víctor Bravo Ahuja No. 125 Esquina Calzada Tecnológico, C.P. 68030; Oaxaca de Juárez, Oaxaca.

ID 1er Autor: Maricela, Morales-Hernández / ORC ID: 0000-0002-3521-2041, CVU CONACYT ID: 731036

ID $1^{\text {er }}$ Coautor: Gabriela, Martínez-Hernández / ORC ID: 0000-0003-4670-7979, CVU CONACYT ID: 1063630

ID $2^{\text {do }}$ Coautor: León, Guzmán-Salas / ORC ID: 0000-0002-4789-3745, CVU CONACYT ID: 1068881

ID $3^{\text {er }}$ Coautor: Eva, Rafael-Pérez / ORC ID: 0000-0003-2793-1254, CVU CONACYT ID: 905268

DOI: $10.35429 /$ JOTI.2020.14.4.17.26

Recibido 20 Julio, 2020; Aceptado 30 Diciembre, 2020

\begin{abstract}
Resumen
El objetivo del presente artículo es presentar la segunda fase del desarrollo de un prototipo de alarma inteligente que coadyuve en la prevención del robo de bicicletas en la Ciudad de Oaxaca, como resultado de un rediseño de un prototipo anterior. En esta segunda fase de la investigación se hicieron mejoras en la autonomía de la alarma, agregando una batería de litio para alimentar la energía del circuito; así también se disminuye el tamaño del circuito cambiando algunos componentes; por otro lado, se desarrolla una aplicación móvil como complemento de las funciones de la alarma. Y, se agrega un gestor de base de datos en tiempo real con el fin de tener los datos de ubicación de la bicicleta en el momento en que se están generando. Finalmente se agrega el trazado de una ruta con ayuda de la aplicación Google Maps, que le permite al usuario enfocar la búsqueda de su bicicleta. En esta segunda fase se continúa trabajando con la metodología de prototipos.
\end{abstract}

Rediseño, Sistema Programable, Prevención de robo

\begin{abstract}
The objective of this article is to present the second phase of the development of a smart alarm prototype that contributed to the prevention of bicycle theft in Oaxaca City, as a result of a redesign of a previous prototype. In this second phase of the investigation, improvements were made in the autonomy of the alarm, adding a lithium battery to supply the energy of the circuit; thus, the size of the circuit is also decreased by changing some components; on the other hand, a mobile application is developed to complement the alarm functions. In addition, a real-time database manager is added in order to have the location data of the bicycle on real time, it means, when the data are being generated. Finally, the traced of a route is added using the Google Maps application that allows the user to focus in certain zone in order to searching their bicycle. In the second phase, also it was applied the prototyping methodology as in the first phase.
\end{abstract}

Redesign, Programmable System, Theft prevention

Citación: MORALES-HERNÁNDEZ, Maricela, MARTÍNEZ-HERNÁNDEZ, Gabriela, GUZMÁN-SALAS, León y RAFAEL-PÉREZ, Eva. Rediseño del prototipo de sistema programable para prevenir el robo de bicicletas en Oaxaca. Revista de Invención Técnica. 2020. 4-14:17-26.

\footnotetext{
*Correspondencia al Autor (Correo Electrónico: moralesh.maricela@gmail.com)

$\dagger$ Investigador contribuyendo como primer autor.
} 


\section{Introducción}

El objetivo del presente artículo es presentar la segunda fase del desarrollo de un prototipo de alarma inteligente, al cual se aplicó un proceso de mejora, agregándole características que mejoran su funcionalidad.

La importancia de mejorar el primer prototipo es darle un valor agregado al mismo, respecto a las alarmas comerciales, minimizando los costos y maximizando las funciones, mediante la utilización de componentes tanto de software como de hardware de mayor calidad y a un costo menor. Además de involucrar a los estudiantes de la carrera de Ingeniería en Sistemas Computacionales del Tecnológico Nacional de México campus Oaxaca, en proyectos que aporten soluciones prácticas a los problemas sociales que se viven actualmente; específicamente, en la Ciudad de Oaxaca de Juárez y sus municipios conurbados, como lo es el robo de vehículos y específicamente de bicicletas, que por ser vehículos ligeros son sensibles a su extracción y venta en el mercado negro.

Resulta pues, como en todas las ciudades en crecimiento, que existe el problema de la inseguridad y se presentan delitos donde los ciudadanos ven perjudicado su patrimonio, como en este caso, una bicicleta, que quizás para muchas personas puede ser un bien con poco valor, para otros representa la oportunidad de desplazarse de un lugar a otro para realizar sus actividades cotidianas; lo cual le da un valor más allá del monetario.

El problema de inseguridad es evidente, de acuerdo con la Encuesta Nacional de Seguridad Pública Urbana (INEGI, 2020), en el mes de septiembre de 2019 la percepción social sobre inseguridad pública arrojó que el $74.4 \%$ de la población de 18 años y más considera que vivir en la ciudad de Oaxaca es inseguro.
Esta percepción se hace patente en los medios masivos de información y se confirma que los robos de bicicleta continúan efectuándose en el municipio de Oaxaca de Juárez, ya que, según el sitio e-consulta.com, el club Ay Nanita Cycling Team fue víctima del robo de una bicicleta en la de la agencia de cinco señores perteneciente al municipio de Oaxaca de Juárez, la unidad robada es una bicicleta de competencia con un valor aproximado de $15 \mathrm{mil}$ pesos; cabe hacer mención que en la misma nota se informa que el club recuperó otra bicicleta que le había sido robada cinco años atrás con un valor de 30 mil pesos (e-oaxaca.com, 2020).

Por lo anterior, se reconoce la urgencia de habilitar a los ciudadanos de herramientas que les permitan salvaguardar sus bienes utilizando las tecnologías emergentes e involucrar en estas soluciones a las instituciones educativas que se encuentran en la ciudad de Oaxaca.

En las empresas que comercializan este tipo de dispositivos se pueden encontrar algunos ejemplares como, por ejemplo: Luz trasera inteligente para bicicleta - Alarma inalámbrica antirrobo recargable, cuyo precio aproximado es de $\$ 1000.00$ pesos mexicanos (Amazon, 2020), o la ANTUSI A8-alarma antirrobo para bicicletas, con freno automático, luz trasera con control remoto para bicicletas de montaña con un costo aproximado de $\$ 409.20$ pesos mexicanos (Aliexpress, 2020). Sin embargo, en ninguno de los casos la alarma cuenta con GPS, los dispositivos que, si tienen incorporada la función de rastreo como lo es WJSW Rastreo GPS a Prueba Agua, 4G GPS con Alarma, tiene un costo aproximado de $\$ 2800.00$ pesos y tiene una aplicación móvil que permite la comunicación con el dispositivo, a través de una aplicación sencilla (Amazon, 2020).

Existen también, otros prototipos experimentales como por ejemplo el que presenta Cabascango en su tesis doctoral, éste prototipo también incluye una aplicación móvil que permite al usuario activar y desactivar funciones de un sistema de alarma, así como también obtener la geolocalización del vehículo, en este caso ha sido diseñado para automóviles (Cabascango, 2020); pero, no cuenta con una base de datos que registre las incidencias. Y, por haber sido diseñado para automóviles, no abarca el segmento de vehículos no motorizados como lo son las bicicletas. 
El prototipo mejorado de la alarma para bicicletas, a diferencia de las opciones comerciales y experimentales citadas en los párrafos anteriores, tiene las siguientes características:

Las medidas del prototipo son $5.75 \mathrm{~cm}$ de largo x $2.70 \mathrm{~cm}$ de ancho x 2.78 de altura. Con lo que se ha reducido su tamaño en un $50 \%$ aproximadamente.

- A diferencia de los anteriores, en este nuevo prototipo se simplificaron las funciones y sólo se implementan tres módulos: SIM, base de datos y cargador de batería de litio.

Funciona con su propia fuente de energía, la cual es una batería de litio que le puede dar un tiempo de autonomía de cuatro horas aproximadamente.

Se ha desarrollado una aplicación móvil que permite rastrear la bicicleta usando la ubicación que se genera en el prototipo y la envía cada cierto tiempo configurable a la aplicación móvil.

El artículo se ha dividido en siete secciones, en las cuales se explican la metodología de desarrollo; el desarrollo, que detalla la forma en que se fueron aplicando los pasos metodológicos; los resultados obtenidos al finalizar esta fase de trabajo; los trabajos futuros donde se plasma la evolución del prototipo obtenido y hacia donde puede continuar la investigación; los agradecimientos a los participantes del proyecto, así como a las instituciones que dieron las facilidades para el desarrollo del mismo; las conclusiones a las que se llega después de un proceso de trabajo y finalmente, la lista de referencias consultadas, que han orientado y fundamentado el presente documento.

\section{Metodología}

La metodología que se utiliza en esta segunda fase del proyecto de investigación es la de prototipos, ya que, es la que ha sido utilizada también en la primera fase.
El término prototipo se deriva etimológicamente según Gengnagel, Nagy y Stark (2016, p.4) de la antigua palabra griega protos, "el primero" y typos, "arquetipo o modelo"; y en la actualidad, desempeña un papel central en los procesos de ingeniería, desarrollo y diseño de todos los campos incluidos en el proyecto.

Una característica principal del prototipo es que las disciplinas donde se aplica reconocen su uso en desarrollos de optimización iterativos y procesos de trabajo en los que cumple diferentes funciones como medio de comunicación y como modelo para el proceso de trabajo inductivo-analítico (Gengnagel, Nagy y Stark, 2016, p.5); esta conceptualización aplica al prototipo que se desarrolla en el presente trabajo, pues se establece que cada prototipo es el resultado de la optimización de las características del anterior.

Como ventajas del uso de la metodología de prototipo se enlistan las siguientes (Barranco, 2001, p.42):

- Facilitar la comunicación entre cliente y analista.

- Permitir al cliente concretar sus necesidades de un modo óptimo.

- Permitir la obtención de resultados visibles en las primeras etapas del desarrollo.

- Disminuir el riesgo de error en la construcción de los sistemas.

- Facilitar la gestión de cambios durante el desarrollo.

Aumentar la productividad del equipo de proyecto.

De forma resumida según Alonso, Martínez y Segovia (2005), se identifican las siguientes etapas en la metodología de prototipos:

Recolección de requisitos.

Diseño rápido.

Construcción del prototipo. 
Evaluación del prototipo.

- Refinamiento del prototipo.

Producto.

\section{Desarrollo}

\section{Requisitos}

Para el tercer prototipo de la alarma inteligente se definen los siguientes requisitos:

Reducción del tamaño aproximadamente en un $50 \%$.

Activación/desactivación de la alarma a través de una tarjeta RFID, la cual tendrá en poder el usuario de la alarma.

- Módulo SIM, el cual enviará las coordenadas de ubicación de la bicicleta a un número de teléfono celular registrado en la programación de la alarma.

Módulo de base de datos, el cual almacena en la base de datos las coordenadas, fecha, hora, entre otros datos que se generan en el sistema programable.

Módulo cargador para pila de litio, el cual permite que el prototipo funcione de forma autónoma aproximadamente de 4 a 6 horas continuas.

Aplicación web, en la cual, utilizando un mapa se visualizan todos los dispositivos que se encuentren activos, se dan de alta a los usuarios de las alarmas, esta opción se habilita pensando en un modelo de negocio donde se rente el servicio de monitoreo de las alarmas.

Aplicación móvil, en la que el usuario pueda activar y desactivar su alarma, registrarse y dar seguimiento a la ubicación de la bicicleta en caso de que ésta haya sido sustraída.

\section{Diseño y construcción}

\section{Módulo SIM}

Este módulo es utilizado para generar la geolocalización del dispositivo; ya que el componente SIM800L de acuerdo con la hoja de datos técnicos es un módulo GSM (Global System for Mobile Communications)/ GPRS (General Packet Radio Service) de cuatro bandas, que funciona en frecuencias GSM850MHz, EGSM900MHz, DCS1800MHz y PCS1900MHz, y con una configuración pequeña de $17.8 * 15.8 * 2.4 \mathrm{~mm}$, es capaz de abarcar un sinfín de aplicaciones que involucren comunicación con teléfonos inteligentes, PDA (Personal Digital Assistant) y otros dispositivos móviles (SIMCom, 2015, p.11).

Para el intercambio de datos entre el módulo SIM y el microcontrolador ATTiny85, se utilizan los comandos AT, según García y Morales son un subconjunto de los comandos Hayes que permiten controlar y configurar los módems desde una computadora o terminal (García y Morales, 2012, p. 171); en este caso, los comandos AT se utilizan para controlar el estado y la configuración de un módem GSM con el fin de enviar la información que genera el GPS (Global Positioning System) que contiene el SIM800L internamente. En la figura 1, se muestra el diagrama a bloques de la conexión entre el SIM800L y el microcontrolador.

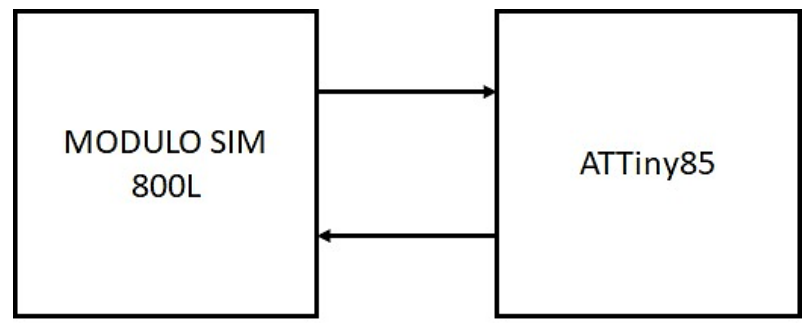

Figura 1 Diagrama a bloques del módulo SIM con el microntrolador

Fuente: Elaboración propia

\section{Módulo base de datos}

En el prototipo anterior se había utilizado MySQL como gestor de la base de datos que almacena la información que se obtiene de la alarma mientras ésta se encuentra en uso; sin embargo, al hacer diferentes pruebas se notó que el intercambio de datos se hacía lento cuando se monta en web. 
Por lo anterior, en este prototipo se utiliza el gestor Firebase, el cual almacena y sincroniza datos con una base de datos NoSQL alojada en la nube. Los datos se sincronizan con todos los clientes en tiempo real y se mantienen disponibles aun cuando la aplicación no tenga conexión (Firebase, 2020). La configuración de comunicación entre diferentes dispositivos y la base de datos se muestra en la figura 2 .

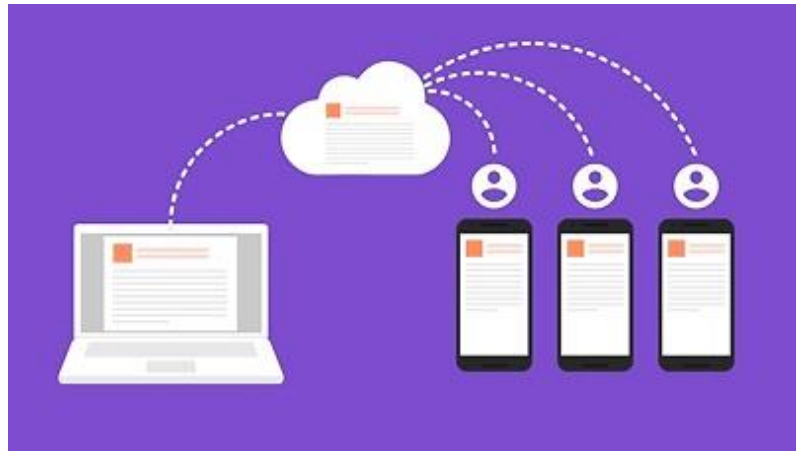

Figura 2 Conexión de base de datos con diferentes dispositivos

Fuente: (Firebase,2020)

\section{Módulo cargador de batería de litio}

Derivado de las mejoras que se aplicaron al nuevo prototipo de la alarma para bicicletas, se le agregó un módulo para carga de baterías; para ello, se utiliza el módulo TP4056 cargador de batería de litio, el cual es un pequeño módulo para la carga de baterías LiPo o Li-ion de una sola celda de $3.7 \mathrm{~V} 1 \mathrm{Ah}$ o superior como las 16550 o las 18650 hay dos versiones de este módulo: sin circuito de protección y con circuito de protección, para que las baterías no reciban ningún daño (Patagoniatec blog, 2020). En este caso el cargador ya incluye el circuito de protección, en la figura 3 se ilustra el componente. El objetivo de agregarle este componente, es con la finalidad de que la alarma para bicicleta pueda ser autónoma en cuanto a la fuente de energía, aspecto que el prototipo anterior no tenía contemplada.

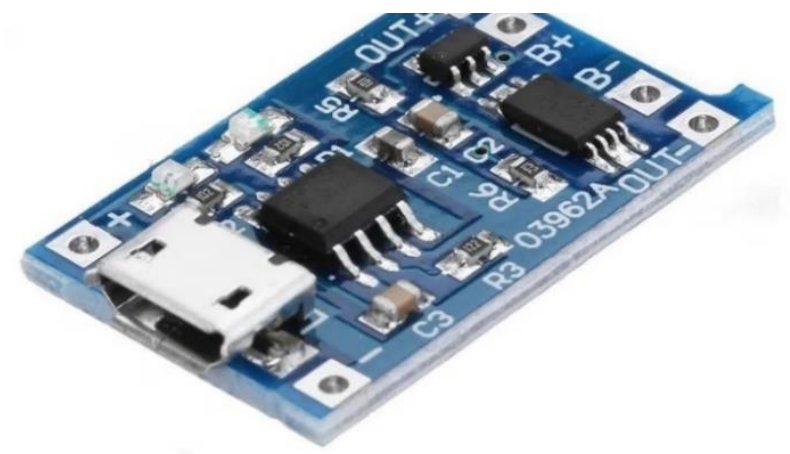

Figura 3 Módulo TP4056

Fuente: (Patagoniatec blog, 2020)
Al cargador se conectó una pila recargable de litio de 3.7 volts a $1500 \mathrm{mAh}$, ésta podrá funcionar de forma continua una vez cargada al $100 \%$ por un lapso de cuatro horas aproximadamente, en la figura 4 se muestra la pila utilizada.

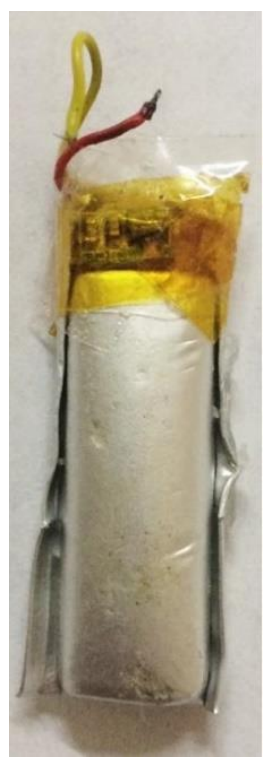

Figura 4 Pila recargable de litio de 3.7 volts a $1500 \mathrm{mAh}$ Fuente: Elaboración propia

En la figura 5 se muestra el diagrama a bloques del módulo cargador de batería, el cual a su vez se conectará a la fuente de recarga externa a través de un puerto USB que viene ya implementado en el circuito.

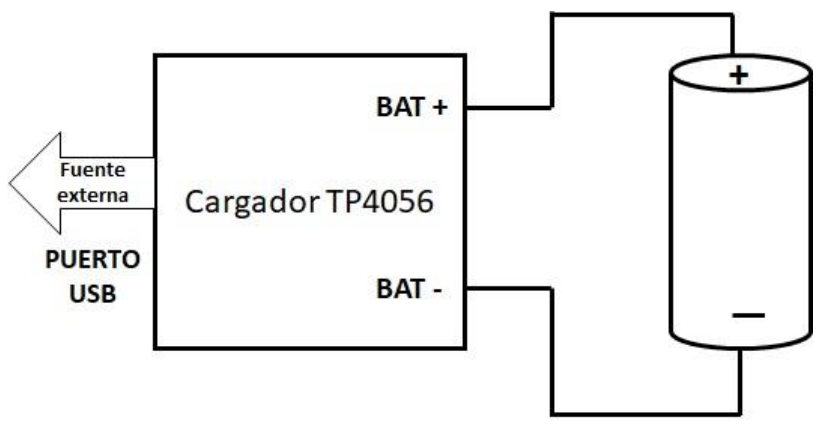

Figura 5 Diagrama a bloques de la conexión entre el cargador de pilas, la pila de litio y la fuente externa de corriente

Fuente: (Elaboración propia)

El diseño del circuito queda como se muestra en la figura 6 , es importante resaltar que los componentes del circuito principal se reducen a dos componentes físicos, y a éstos se le agrega la parte de la fuente de alimentación que es una pila recargable con el circuito que permite la recarga de la pila. Con ello, se logra reducir aproximadamente el 50\% el tamaño del circuito en relación con el prototipo inicial. 


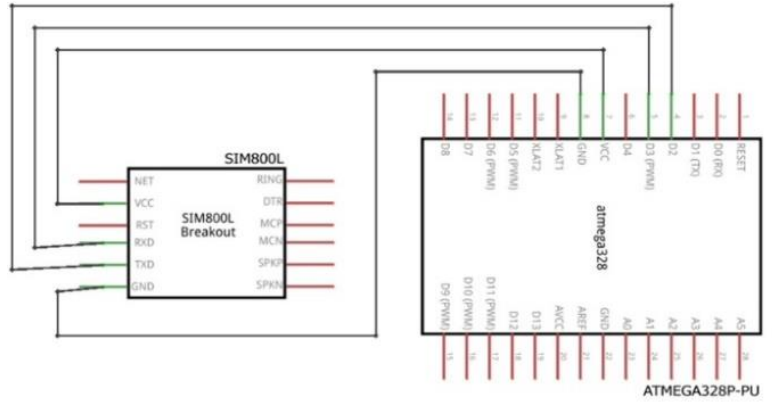

Figura 6 Diseño físico del circuito final Fuente: (Elaboración propia)

\section{Aplicación web}

La aplicación web de la alarma se diseña y construye como una opción donde se ofrezca el servicio de monitoreo de alarmas a diferentes clientes, planteando que adquieran éstos una membresía que les permita tener acceso a herramientas complementarias a la alarma. El diseño de una aplicación web se realizó por sugerencia de usuarios; ya que el prototipo se presentó en foros de proyectos tecnológicos, tales como el Evento Nacional Estudiantil de Innovación Tecnológica fases local y regional; así como Proyecto Multimedia, Ciencia y Tecnología, sede regional Oaxaca.

En la aplicación web se planean dos tipos de roles: administrador y usuario de la alarma. El primero tiene acceso a la información de todos los usuarios de la alarma, como también la ubicación de los dispositivos en tiempo real. El segundo es el usuario que adquiere una alarma para su bicicleta y también puede revisar los desplazamientos que ha hecho el vehículo y que se encuentran registrados en la base de datos. También se considera que en la aplicación web se consulten los datos almacenados en tiempo real en la base de datos firebase.

\section{Aplicación móvil}

Debido a que en el segundo prototipo que se obtuvo en la investigación, se contempla el envío de mensajes SMS al número del teléfono móvil registrado y también considerando las observaciones que se hicieron al prototipo, cuando fue exhibido en los foros de ciencia citados en párrafos anteriores, se planea el diseño de una aplicación móvil que permita al usuario ingresar con un nombre y contraseña, y activar el prototipo para que éste sea visible en la aplicación web.
Así también el usuario de la alarma tiene una opción para ver la ubicación de su bicicleta en tiempo real. Esta aplicación móvil está enlazada al prototipo por medio de la información que se envía a través del módulo SIM.

\section{Herramientas de programación}

Para lograr que la alarma tenga las funcionalidades descritas en el apartado 1.1 (Requisitos), se utilizan diferentes herramientas para su programación; así, para el microcontrolador ATTiny85, al ser de la familia AVR es posible programarlo con el IDE para Arduino, el cual se basa en C++. Por lo que la programación es similar a la de una placa Arduino.

Por otro lado, la aplicación móvil se programa en Android Studio, cuya base es el lenguaje Java. Y la página web utiliza en su programación el framework Booststrap, ya que dentro del mismo se pueden tener herramientas como hojas de estilo en cascada (CSS), lenguaje PHP, JavaScript, entre otras (Bootstrap, s.f.).

\section{Evaluación del prototipo}

Para evaluar el prototipo se aplicaron diferentes pruebas; primero, se hicieron pruebas de cada módulo para verificar su funcionamiento de forma independiente, después se aplicaron pruebas de integración para revisar las funcionalidades en conjunto, se encontraron algunos detalles en la programación del microcontrolador; sin embargo, se corrigieron en su momento para obtener la funcionalidad que se había planificado.

Una vez que los componentes de hardware fueron probados, se probó la funcionalidad del software; en este caso se probó primero la comunicación entre el prototipo y el celular a través de mensajes SMS, comprobando que no existía problema, se continuó probando las funciones de la aplicación móvil, detectando detalles con la activación de la alarma, los errores se solucionaron y algunas funciones se mejoraron, como por ejemplo: los datos que se almacenan en la base de datos, se agregaron algunos que permitan identificar de forma única cada registro. 
Finalmente se probaron tanto software como hardware del prototipo, sometiéndolos a pruebas integrales, obteniendo los resultados esperados después de realizar algunos ajustes.

\section{Refinamiento del prototipo}

El prototipo obtenido en este trabajo denominado Alarma Inteligente para prevenir el robo de bicicletas en la ciudad de Oaxaca, tiene aspectos que pueden ser mejorados en trabajos de investigación futuros, que implican la incursión de nuevas tecnologías de programación y un modelo de negocio para que pudiera ser comercializado cuando el prototipo haya alcanzado cierta madurez; así mismo la parte del hardware puede ser mejorada en el aspecto de tener una fuente de energía renovable y libre de contaminación, como puede ser la energía solar, o la energía generada por el usuario de la bicicleta (por fricción), al momento de estarla utilizando. Otro aspecto que se puede seguir mejorando es la miniaturización del dispositivo de tal forma que, éste pueda ser oculto en los tubos del cuadro principal de la bicicleta sin que los delincuentes puedan notar su ubicación. En resumen, los trabajos futuros estarían enfocados a:

- Hardware más pequeño y con las mismas o mejores funcionalidades.

- Programación con nuevas tecnologías emergentes que permitan usar diferente generación de protocolos de comunicación.

- Fuente de energía renovable y amigable con el ambiente.

Modelo de negocio que sea sustentable y dé pie a la incubación de una empresa con tecnología y marca propia.

\section{Resultados}

Los resultados obtenidos se integran en un nuevo prototipo de alarma inteligente para prevenir el robo de bicicletas, este prototipo consta de los siguientes componentes:

Sistema programable con pila recargable que controla la localización de la bicicleta.
- $\quad$ Página web.

El sistema programable final se presenta en la figura 7 , sus dimensiones son $5.75 \mathrm{~cm}$ de largo por $2.70 \mathrm{~cm}$ de ancho por 2.78 de altura, lo cual permite que sea colocado en una parte donde no sea visible.

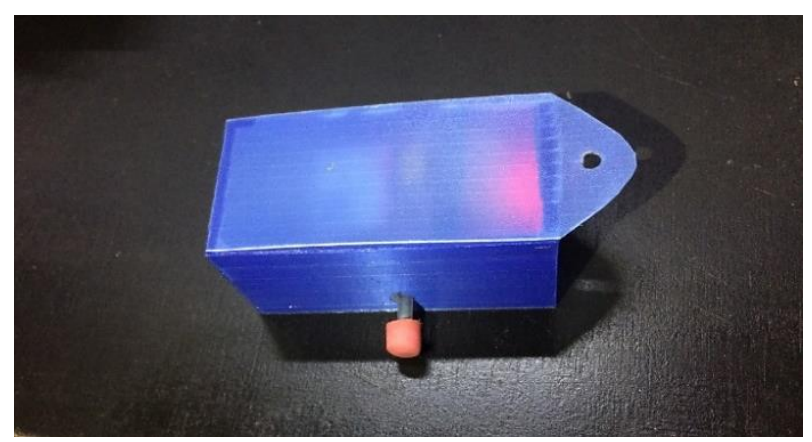

Figura 7 Prototipo de la alarma inteligente Fuente: (Elaboración propia)

Al ser el prototipo de pequeñas dimensiones, puede colocarse debajo de la bicicleta, entre los tubos del asiento, como se ilustra en la figura 8 .

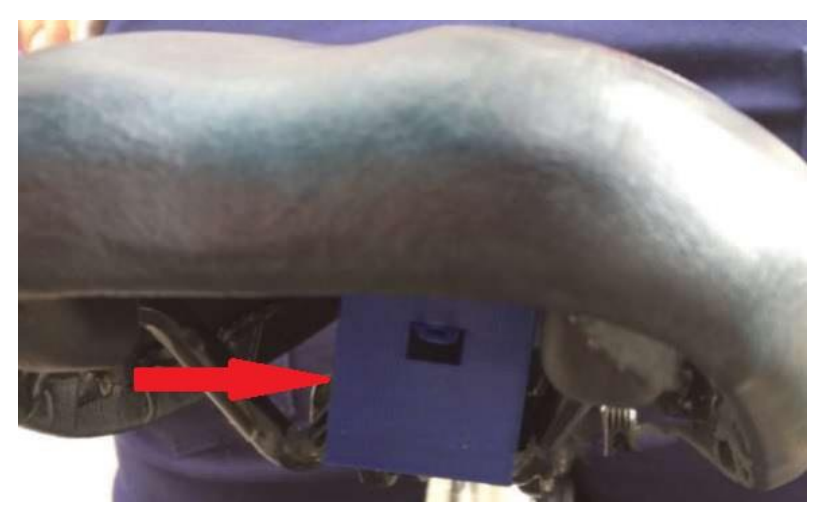

Figura 8 Instalación del sistema programable Fuente: (Elaboración propia)

La alarma también cuenta con un botón de encendido/apagado como puede verse en la figura 9. Para encenderlo basta con presionar y después soltar el botón, y la misma acción se ejecuta para apagarlo. La aplicación móvil se copia al dispositivo móvil (previa configuración para que acepte aplicaciones de otras fuentes), y desde allí se instala. Una vez instalada y ejecutándose, se presentan dos opciones, como se ilustra en la figura 10. La primera opción permite que el usuario se registre para enlazar el equipo cuando es nuevo; y la segunda, permite iniciar sesión cuando ya está registrado el usuario.

- $\quad$ Aplicación móvil. 


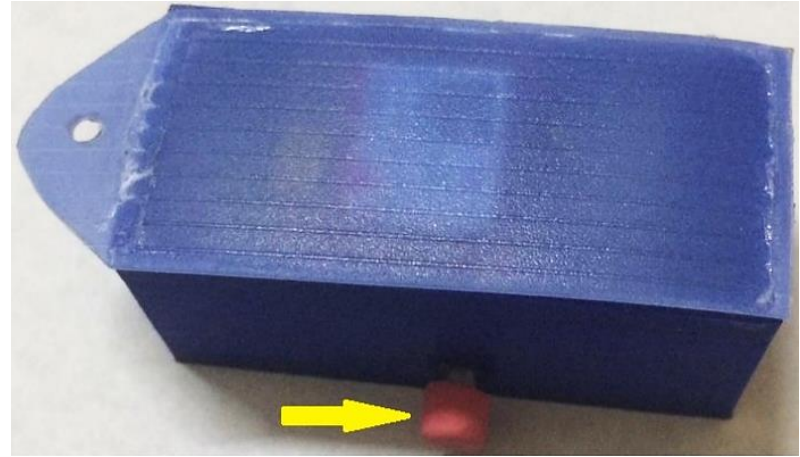

Figura 9 Botón de encendido/apagado Fuente: (Elaboración propia)

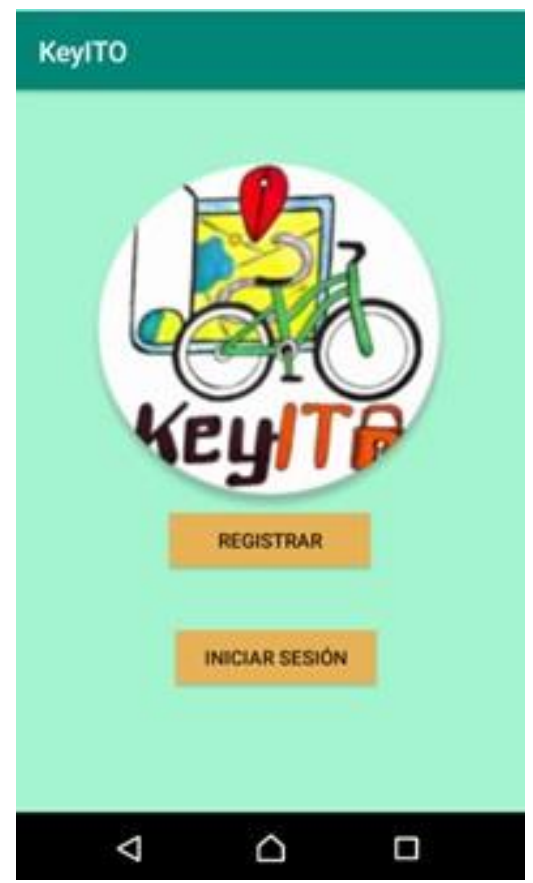

Figura 10 Pantalla principal de la aplicación móvil Fuente: (Elaboración propia)

Una vez iniciada la sesión del usuario, éste cuenta con dos opciones dentro de la aplicación, como se ve en la figura 11 , se le permite ver la ubicación actual de la bicicleta, o bien, cerrar la sesión. Una vez activo el dispositivo, es decir, cuando se presenta una alerta, al seleccionar la opción VER UBICACIÓN DE LA BICICLETA se muestra un mapa, el cual va dando seguimiento a la ruta que sigue la bicicleta si está siendo movida del lugar donde se deja originalmente.

En la figura 12 puede verse un ejemplo de una ruta trazada, este experimento se realiza para probar la correcta funcionalidad del prototipo. Se observa que la bicicleta ha sido llevada desde la Ciudad de Oaxaca hasta otra ubicación relativamente lejana, pero ubicable, con ello se reafirma que el prototipo funciona como estaba planificado.

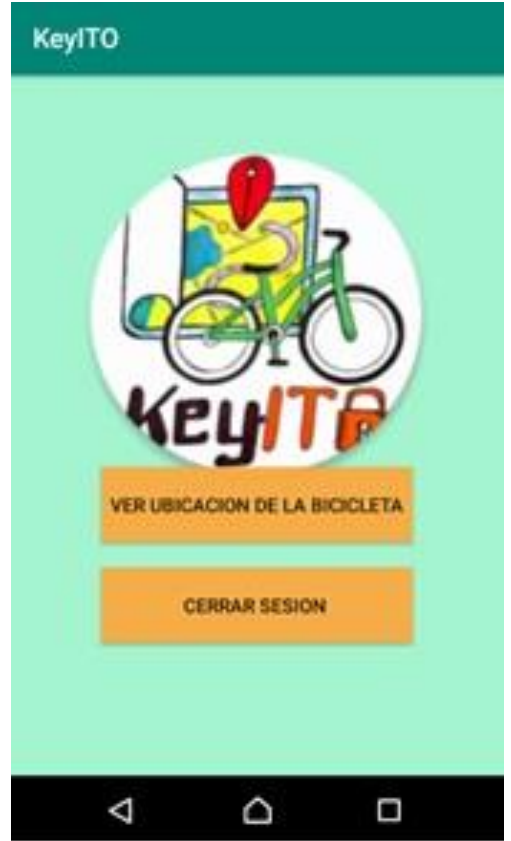

Figura 11 Opciones del usuario de la aplicación móvil Fuente: (Elaboración propia)

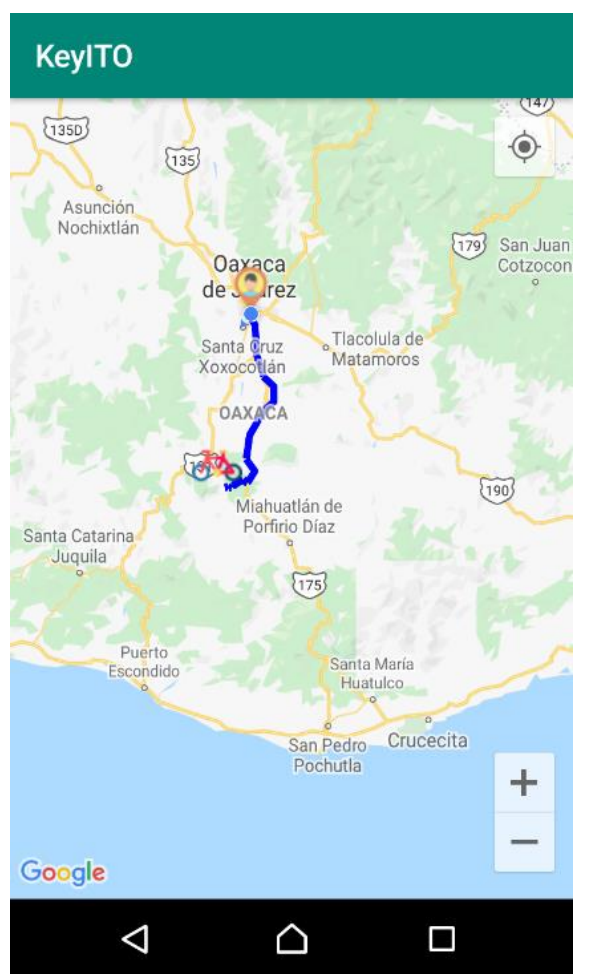

Figura 12 Ruta trazada del seguimiento de la bicicleta Fuente: (Elaboración propia)

La página web contiene dos vistas: la del usuario y la del administrador. En la vista del administrador la autenticación se efectúa a través de un correo electrónico y contraseña, como se muestra en la figura 13, también contiene una opción de recuperación de contraseña en caso de que sea olvidada o extraviada. El administrador ingresa a la página principal y tiene el acceso a un mapa con la ubicación de todos los dispositivos registrados, tal como puede verse en la figura 14. 

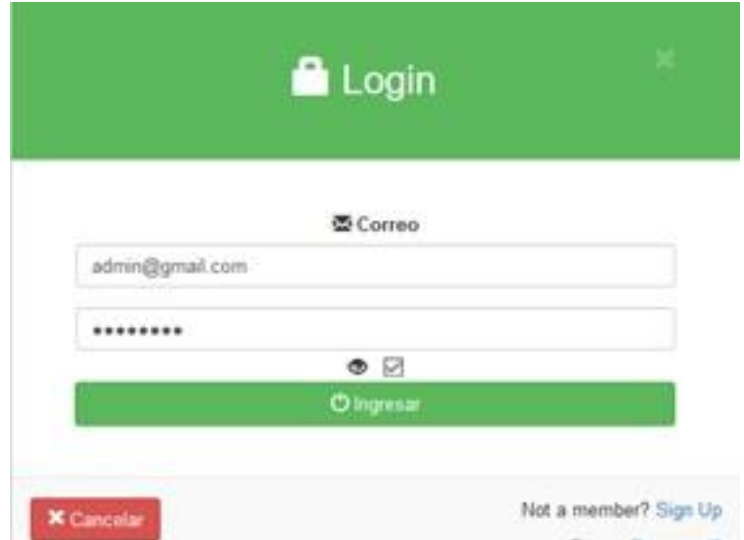

Not a member? Sign Up Forgot Pasmenen?

Figura 13 Vista de inicio de sesión de la página web Fuente: (Elaboración propia)

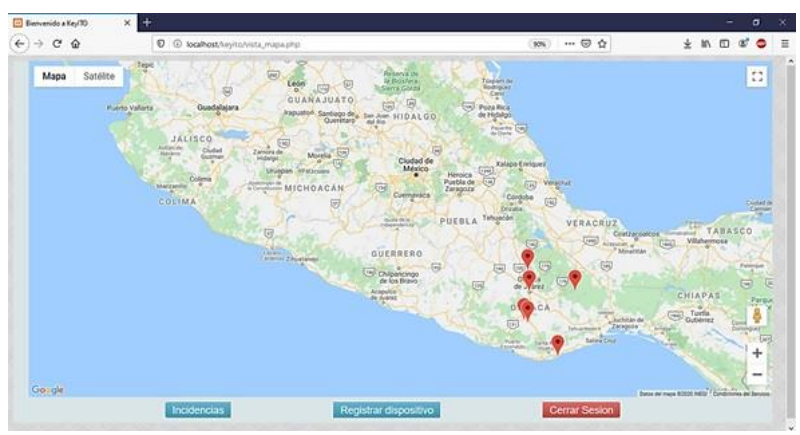

Figura 14 Mapa con la ubicación de los dispositivos activos registrados

Fuente: (Elaboración propia con apoyo de la herramienta Google Maps)

La vista del usuario de la página web de forma similar a la del administrador, tiene un proceso de autenticación basado en el correo del usuario y una contraseña, y la ventana es la misma ilustrada en la figura 13, es importante hacer hincapié que el usuario se registra previamente y si en un momento dado olvida su contraseña, puede recuperarla. Pero, a diferencia de la vista administrador en el mapa solo se muestra la ubicación de su dispositivo, esta idea se ilustra en la figura 15.

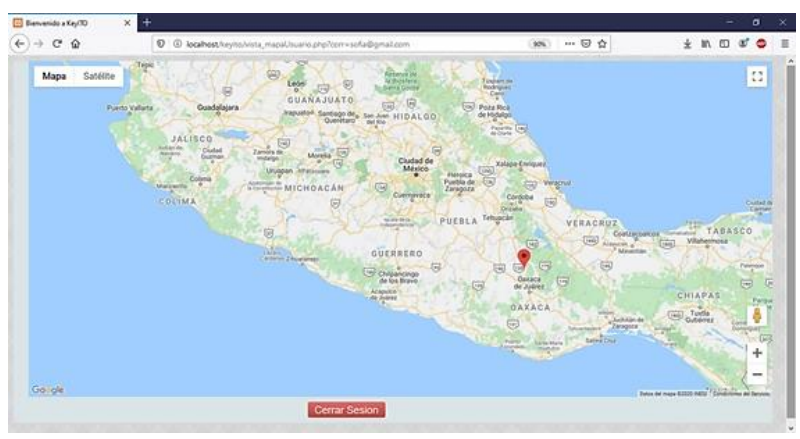

Figura 15 Ubicación actual del dispositivo en la vista del usuario

Fuente: (Elaboración propia con apoyo de la herramienta Google Maps)

\section{Agradecimientos}

Al Tecnológico Nacional de México se agradece que, a través del Campus Oaxaca, ha dado las facilidades y los espacios para el desarrollo del presente trabajo de investigación.

También se agradece enormemente la colaboración, la paciencia y dedicación de los autores del artículo, profesores y estudiantes que han participado en la investigación hasta lograr los resultados que se presentan; cuyo objetivo es divulgar los hallazgos a la comunidad académica y al público en general sobre los trabajos que se desarrollan en las instituciones educativas.

\section{Conclusiones}

El prototipo resultante ha permitido encontrar nuevas oportunidades de mejora, y la metodología basada en prototipos permite ir desarrollando el dispositivo a través de iteraciones donde se repite el proceso y se va construyendo un sistema cada vez más completo y apegado a las necesidades reales del usuario final.

En este nuevo prototipo se tienen complementos que aplicando las tecnologías emergentes permiten dar un seguimiento a la bicicleta, no solo en caso de que ésta haya sido sustraída; sino, incluso, para dar seguimiento a un recorrido del usuario, cuando éste se siente vulnerable al conducir solo por algún sendero o recorrido que impone cierto riesgo.

El costo del prototipo aún no es preciso; sin embargo, haciendo un resumen de los tres prototipos desarrollados durante la investigación, el costo fue variando de $\$ 1,000.00$ pesos mexicanos el 1er prototipo, $\$ 800.00$ pesos mexicanos el segundo prototipo y $\$ 500.00$ pesos mexicanos el tercer prototipo; éste último es el que se presenta en este documento. Este costo todavía puede reducirse, pues se contempla la posibilidad de mejorar aspectos como la miniaturización y la fuente de energía del sistema de alarma con el fin de que sea alimentado por un tipo de energía renovable y amigable con el ambiente. 
Por otro lado, en las presentaciones que se llevaron a cabo del prototipo en foros de innovación tecnológica, con las sugerencias de los evaluadores y público en general, se ha encontrado la oportunidad de iniciar un modelo de negocios donde se preste el servicio de monitoreo, semejante al que usan las empresas que cuentan con una flotilla de vehículos para distribución de productos o servicios; con este servicio se pueden tener usuarios con sus dispositivos, pagando una membresía. Para las empresas representaría una forma de evaluar la efectividad de sus rutas, la eficiencia de sus empleados, las rutas libres de bloqueos, entre otros; ya que la ciudad de Oaxaca frecuentemente presenta este tipo de incidencias. Finalmente, la investigación en los centros de educación superior, representan un gran impacto en el área de influencia de estos, pues desde la formación de los profesionales, éstos se involucran con problemas reales de su entorno, permitiendo que, a través de la creatividad y aplicación del conocimiento, se presenten soluciones reales y factibles de ponerse en marcha a un precio justo para el usuario final, quien requiere de protección para su patrimonio, en este caso, la bicicleta.

\section{Referencias}

Aliexpress (2020). Accesorios de bicicletas: Alarmas antirrobo. Consultado el 13 de julio de 2020. https://es.aliexpress.com/

Alonso, F., Martínez, L. y Segovia, F.J. (2005). Introducción a la Ingeniería del software. España: Delta Publicaciones.

Amazon (2020). Departamento de electrónicos: Alarmas para bicicletas. Consultado el 13 de julio de 2020. https://www.amazon.com.mx/

Barranco, J. (2001). Metodología del análisis estructurado de sistemas. España: Universidad Pontificia Comillas Madrid.

Boostrap. (s.f.). Getting Started: Introduction. Consultado el 15 de julio de 2020. https://getbootstrap.com/docs/4.5/gettingstarted/introduction/
Cabascango, J.E. (2020). Diseño e implementación de alarma vehicular con geolocalización, mediante el uso de aplicaciones móviles [tesis de licenciatura, Universidad Técnica del Norte, Ibarra, Ecuador]. Repositorio Digital Institucional. http://repositorio.utn.edu.ec/handle/123456789/ 10413

e-oaxaca.com. (2020, 5 de junio). Roban bicicleta a integrante del Club ¡Ay Nanita!. Econsulta.com Oaxaca. http://e.oaxaca.com

Firebase. (2020). Productos: Cloud Firestore. Consultado el 15 de julio de 2020. https://firebase.google.com/products/firestore

García, J. y Morales, G. (2012). Instalaciones de radiocomunicaciones. $1^{\mathrm{a}}$ edición. España: Paraninfo ediciones S.A.

Gengnagel, C., Nagy, E. y Stark, R. Rethink! Prototyping: Transdisciplinary Concepts of Prototyping. Switzerland: Springer.

Instituto Nacional de Estadística, Geografía e Informática. (2020, 16 de enero). Encuesta Nacional de Seguridad Pública Urbana. Comunicado de prensa Núm. 013/20. https://www.inegi.org.mx/programas/ensu/defa ult.html

Patagoniatec blog. (2020). Módulo TP4056 Cargador De Batería. Consultado el 15 de julio de 2020 . https://saber.patagoniatec.com/2019/09/modulo -tp4056-cargador-de-bateria/

SIMCom. (2015). SIM800H \& SIM800L Hardware Design V2.02. China: SIMCom. 